Axel Beer (Mainz)

\title{
Zum Sinn und zum Stand der Erforschung des musikalischen Verlagswesens
}

Was Georg Knepler sich bei der Formulierung dachte, Beethoven hätte zu einem gewissen Zeitpunkt den „Kampf gegen die Verleger [...] weitgehend gewonnen“, ${ }^{1}$ erschließt sich nicht vollständig. Zweifellos aber wählte er das Bild bewusst - nicht nur er sah Beethoven fortwährend in Kämpfe unterschiedlicher Art verwickelt ${ }^{2}$ - und bediente hiermit einerseits den über die ideologischen Lager hinweg gängigen Topos des Heroentums des Meisters, andererseits auch die ebenso gängige Vorstellung von einem Berufsstand und Gewerbezweig, der bar jeder Einsicht der Hervorbringung und freien Entfaltung künstlerischer Leistungen beständig im Weg steht: Musikverleger eben - ahnungslose Schacherer, die um ihrer schnöden Geldgier willen hohes Geistesgut und humanistische Ideale zur Ware umbiegen, mithin deren Schöpfer und Urheber (denen zudem das romantische Künstlerbild eine pragmatisch orientierte Verankerung im Diesseits nicht zugesteht) nach Strich und Faden betrügen. Zugegeben - die in beträchtlicher Zahl überlieferten Äußerungen namhafter wie auch weniger bekannter Komponisten über ihre vom Musikalienhandel lebenden Zeitgenossen scheinen jenes Urteil zu bestätigen; ${ }^{3}$ sie tun dies dann, wenn man sie aus dem Zusammenhang löst und immer aufs Neue geradezu genussvoll zitiert, daraus Folgen zieht bzw. einfach nur wertende Behauptungen aufstellt ${ }^{4}$ oder, wie Knepler es für richtig hielt, durch Verallgemeinerungen in knappster und geradezu apodiktischer Form einen Gegensatz von „Gut“ und „Böse“ konstruiert, folglich das Thema im Grunde als eines wissenschaftlichen Kontexts unangemessen empfindet. Teilt man diesen Standpunkt, so erübrigt sich die ansonsten zu Recht postulierte Exaktheit der Darstellung - wer diese Verleger im Einzelnen waren, welche Beziehung etwa Beethoven (mit welchen Folgen) tatsächlich zu ihnen unterhielt, war nicht nur für Knepler kaum einer Überlegung wert. Und wenn letzterer, selten genug, tatsächlich einen Vertreter dieser Spezies nennt, geht der Griff mitunter knapp, aber entscheidend daneben: Franz Anton Hoffmeister ist nicht identisch mit Friedrich Hofmeister, ${ }^{5}$ was auch Carl Dahlhaus hätte wissen können (und sollen), dem

1 Georg Knepler, Musikgeschichte des 19. Jahrhunderts, Bd. 2: Österreich, Deutschland, Berlin 1961, S. 576.

2 Auf die weltanschauliche Komponente im Denken Kneplers sei hier nicht eingegangen, nur beiläufig darauf verwiesen, dass der Begriff „Klassenkampf“ selbstverständlich im Register des genannten, im Übrigen gleichwohl nach wie vor mit Gewinn zu lesenden Werks ausgeworfen ist.

3 Vgl. hierzu Axel Beer, Musik zwischen Komponist, Verlag und Publikum. Die Rahmenbedingungen des Musikschaffens in Deutschland im ersten Drittel des 19. Jahrhunderts, Tutzing 2000, S. 155-157.

4 Ein „schönes“ (unter vielen möglichen ausgewähltes) Beispiel bietet eine Formulierung über das Tun Tobias Haslingers, das man unter Benutzung einer anderen Brille auch als weitsichtige und für den Gang der Musikgeschichte folgenreiche Rettungstat hätte interpretieren können: „Nach Schuberts Tod raffte er alles an sich [...] “; s. Artikel „Verleger“, in: Schubert-Enzyklopädie, hrsg. von Ernst Hilmar und Margret Jestremski (= Veröffentlichungen des Internationalen Franz Schubert Instituts 14), Bd. 2, Tutzing 2004, S. 791-793, hier: S. 791.

5 Knepler, Musikgeschichte des 19. Jahrhunderts (wie Anm. 1), S. 970, Anm. 625 zu S. 545: Hofmeister (damals knapp 19 Jahre alt) wird hier irrtümlich als Adressat eines von Beethoven an Hoffmeister ge- 
die inzwischen geradezu zum „Klassiker“ gewordene Verwechslung in seinem Buch Beethoven und seine Zeit nicht nur mehrfach, sondern ausnahmslos unterlief. ${ }^{6}$ Jene "Zeit"-Sicht erlaubte offenbar nur die ernsthafte Berücksichtigung derjenigen Gegenstände, Personen und Ereignisse, die des Meisters als würdig erscheinen - folglich auch des Verfassers, der sich mit letzterem in geistiger Verwandtschaft, gleichsam auf Augenhöhe, wähnt.

Vielleicht sollte man sich der Tatsache erneut und mit allen nicht uninteressanten Konsequenzen bewusst werden, dass der weitaus größte Teil des Beethoven'schen Schaffens zu Lebzeiten des Komponisten gedruckt und somit veröffentlicht wurde. Dabei waren es nicht etwa gewissenlose und raffinierte Geschäftemacher, die sich heimlich und aus niederen Beweggründen über den sorgfältig bewahrten Schatz an Manuskripten hermachten. Wäre dem so gewesen (und manchmal scheint es, dass man es glauben soll), so würde der Hohensteiner Kantor Christian Gotthilf Tag (1735-1811) geradezu als Idealbild des Komponisten anzusehen sein, weiß Robert Eitner doch - unter Zuhilfenahme eines für ihn untypischen, aber denn doch allzu verführerischen Wortspiels - zu berichten: „Tag komponierte Tag für Tag u. seine Schränke strotzten voll eigener Werke; trotzdem fühlte er nie das Bedürfnis davon der Welt etwas mitzuteilen, und was bekannt geworden ist, geschah ohne seine Absicht. Er schrieb nur für sich und dem eigenen Drange folgend und fühlte vollkommene Befriedigung in sich selbst; dabei eingeengt in die kleinsten dürftigsten Verhältnisse."7 Was Eitner nicht wissen konnte, da er keine Kenntnis hatte vom umfangreichen Archiv des Musikverlags C. F. Peters (heute verwahrt im Sächsischen Staatsarchiv in Leipzig $\left.^{8}\right)$ : Der Kantor entsprach keineswegs jener hübschen Schilderung seiner vermeintlich romantischen Künstlernatur, denn ganz pragmatisch bot er, wie die allermeisten der Zeitgenossen ebenfalls, seine Werke zur Inverlagnahme an, hoffend, dass er ein seine „dürftigen Verhältnisse“ milderndes Honorar erhalten würde; ${ }^{9}$ eine Geschäftsverbindung kam in diesem Falle jedoch nicht zustande, da die „Stecherei“ des angesprochenen Verlags mit anderen Ausgaben „überhäuft“ war: ${ }^{10}$ Das Leipziger Bureau de Musique hatte unmittelbar nach der Erstveröffentlichung der Opera 19-22 von Beethoven reichlich Altlasten in Form rückständiger Pränumerationsausgaben (Bach und Mozart) zu bewältigen. Das kleine Beispiel mag verdeutlichen, wie schnell die Träger bekannter und weniger bekannter Namen, unter ihnen eben auch Verleger, bei der Betrachtung der Quellen (nicht nur) zum Musikverlagswesen unweigerlich zusammenrücken, was den Blick freigibt auf die eigentlichen,

richteten Briefs bezeichnet; im Register scheint Hofmeister (mit Hinweis auf die genannte Stelle) ohne Vornamen auf, während Hoffmeister als Komponist von Flötenwerken Erwähnung findet, nicht aber als der mit Beethoven in enger Beziehung stehende Verleger.

6 Carl Dahlhaus, Beethoven und seine Zeit, Laaber 1987, S. 13, 50-51, 55, 192. Weitere Belege würden etliche Seiten füllen; vgl. hierzu Beer, Musik zwischen Komponist, Verlag und Publikum, S. 3-4, sowie auch ders., Das Leipziger Bureau de Musique (Hoffmeister \& Kühnel, A. Kühnel). Geschichte und Verlagsproduktion (1800-1814), Einleitung (Druck in Vorbereitung).

7 Robert Eitner, Artikel „Tag, Christian Gotthilf“, in: Biographisch-bibliographisches Quellenlexikon, Bd. 9, Leipzig 1903, S. 342-344, hier: S. 342.

8 Vgl. hierzu Thekla Kluttig, „Nur Briefe berühmter Komponisten? Archivgut von Leipziger Musikverlagen als Quelle für die Musikwissenschaft", in: Mf66 (2013), S. 391-407.

9 Briefe von Christian Gotthilf Tag an das Leipziger Bureau de Musique (Hoffmeister \& Kühnel), Hohenstein 3. Jan. und 23. Nov 1802; Sächsisches Staatsarchiv Leipzig, Bestand 21070 Musikverlag C. F. Peters, innerhalb Nr. 2594.

10 Das Bureau de Musique an Christian Gotthilf Tag, Leipzig 15. Dez. 1802, zit. nach dem Briefkopierbuch 1802/03, Sächsisches Staatsarchiv Leipzig, Bestand 21070 Musikverlag C. F. Peters Nr. 5022, S. 202. 
von selektiven Maßnahmen späterer Zeit unangetasteten historischen Gegebenheiten. Und da Beethoven schon zum Beginn seiner Karriere als Musiker und Komponist nicht dem von Eitner gemalten Bild des sächsischen Kantors entsprechen sollte, vermittelte sein Lehrer Christian Gottlob Neefe (nebenbei: ein Schüler Tags) erste Kompositionen des Knaben (WoO 47-49, 63, 107 und 108) an die Verleger Boßler in Speyer und Götz in Mannheim. Hiermit wollte er ihn ebenso wenig ins offene Messer ahnungsloser Schacherer laufen lassen wie Leopold Mozart, der seinem Sohn riet, in Paris „mit einem graveur“ zu reden und dabei zu erkunden, was dieser „am liebsten haben möchte.“11 In beiden Fällen ging es darum, die jungen Komponisten ,in die große Musikwelt"12 einzuführen, was nichts anderes als eine existentielle Notwendigkeit war. Freilich kann man es auch anders sehen und postulieren, dass ein Komponist, der „sein Werk ein für allemal einem Verleger“ überlässt, „in vorkapitalistischen Strukturen stecken [bleibt], Lohndiener gleichsam, ausgebeuteter Lakai“"13 - nicht auszudenken, was geschehen wäre, wenn alle Tonsetzer sich der Ausbeutung widersetzt und das heute unverzichtbare Kulturgut einfach nur in ihre Schränke gestopft hätten ...

Die Marginalisierung des Musikverlagswesens inklusive seiner Geschichte und mitunter seine Verächtlichmachung im musikhistorischen Schrifttum haben natürlich eine Ursache, die vor allem im nach außen getragenen Selbstbild einer sich um universitäre und öffentliche Anerkennung und Etablierung bemühenden Fachdisziplin zu suchen ist. Eine im 19. Jahrhundert noch junge Wissenschaft konnte und wollte sich nicht mit Dingen auseinandersetzen, deren Kunstcharakter (und somit auch Tauglichkeit für allerlei Identifikationsprozesse) durch das Eingeständnis hätte in Frage gestellt werden können, dass es sich beim Untersuchungsgegenstand (auch) um eine Ware, um Konsumgut handelt. Im Blick auf den Ursprung mag man Verständnis aufbringen für jenen Versuch einer strategischen Rechtfertigung, während die Beständigkeit der Anschauung mit dem Gipfelpunkt in Adornos hinreichend bekannten Thesen und deren ebenso bekannten Folgen Rätsel aufgibt. Geradezu scheint es, dass eine Schweigespirale in Gang gesetzt wurde, die trotz gelegentlicher Vorstöße $\mathrm{e}^{14}$ nicht zu durchbrechen ist und die einem geschärften Bewusstsein hinsichtlich des möglichen Erkenntnisgewinns aus dem einschlägigen, vielfältigen und nach wie vor wenig bis gar nicht genutzten Quellenmaterial im Wege steht. Erweiterte Fragestellungen, die etwa Distributionsmechanismen, Geschmacksbildung unter Berücksichtigung unterschiedlicher geographischer und soziologischer Gegebenheiten, Verdrängungsprozesse von Musizierformen und musikalischen Traditionen zu betrachten hätten, könnten umfassenden Gewinn erbringen. Jedoch fristen verlagshistorische Fakten im Allgemeinen ein Fußnotendasein; allenfalls die Referenzliteratur - Werkverzeichnisse, Kritische Berichte u. a. listet sie mit buchhalterischer Sorgfalt auf, und zwar im Rahmen dessen, was bisher er-

11 Leopold Mozart an Wolfgang Amadé Mozart, Salzburg 13. Aug. 1778, zitiert nach: Mozart. Briefe und Aufzeichnungen. Gesamtausgabe, hrsg. von Wilhelm A. Bauer und Otto Erich Deutsch, Bd. II: 1777-1779, Kassel u. a. 1962, S. 442-447, hier: S. 444.

12 Eucharius Florschütz an das Leipziger Bureau de Musique (Hoffmeister \& Kühnel), Rostock 31. Juli 1803; Sächsisches Staatsarchiv Leipzig, Bestand 21070 Musikverlag C. F. Peters, innerhalb Nr. 2595.

13 Christian Kaden, Artikel „Musiksoziologie“, in: MGG2, Sachteil 6, Kassel u. a. 1997, Sp. 1618-1670, hier: Sp. 1634.

$14 \mathrm{Vgl}$. (neben den genannten und weiter unten zu nennenden Arbeiten) zuletzt Laurenz Lütteken, „Musikalisches ,am Markt‘. Typologische Überlegungen zu einer kulturellen Praxis im 18. Jahrhundert“, in: Das achtzehnte Jahrhundert 36 (2012), S. 258-270. 
mittelt wurde. Da dies alles andere als ausreichend ist, finden sich auch dort Irrtümer, Versäumnisse und Fehleinschätzungen, wo man eigentlich eine gesicherte und belastbare Materialbasis zu erwarten hat: Mangelnde Datierungsmöglichkeiten auf Grund fehlender Grundlagenforschung, daraus resultierend die oft genug begegnende Unmöglichkeit einer Hierarchisierung einzelner Ausgaben, zudem die noch immer nicht einheitliche, bisweilen irreführende Terminologie (Erst- und Originalausgabe, Auflage, Titelauflage, Neuausgabe, Nachdruck, Übernahme u. a. ${ }^{15}$ ) lassen mitunter Folgerungen aus dem erhobenen Faktenbefund nur allenfalls mit Mühen zu. ${ }^{16}$

Der in der Forschung etablierte, auf Grund eines breiten Methodenschatzes in vielfältiger Weise deutende Ansatz scheint bibliographische und verlagshistorische Befunde, die im günstigen Falle einer zielführenden Fragestellung unterzuordnen sind, kaum zu benötigen. Da Grundlagenforschung - mehr oder weniger deutlich ausgesprochen - bisweilen in die Nähe des biederen, zwar nicht verwerflichen, aber der Wissenschaft vermeintlich nicht zuträglichen Positivismus (gerne ist unter vorgehaltener Hand von „Datenhuberei“ die Rede) gerückt wird, sucht man ihre Spuren im „offiziellen“ Forschungs- und Lehrbetrieb (fast) vergeblich. Symptomatisch ist die Tatsache, dass eine in den 1960er Jahren im Zuge der RISM-Arbeiten entstandene Initiative zur Erforschung der Geschichte des Musikverlagswesens nach nicht langer Zeit einschlief; ${ }^{17}$ ebenso bezeichnend ist es, dass eine in den 1990 er Jahren von der European Science Foundation unterstützte, international besetzte Arbeitsgruppe bislang zwar beachtliche Ergebnisse zum Thema Circulation of Music vorlegen konnte, ${ }^{18}$ von denen wahrnehmbare Impulse hingegen kaum ausgingen. Schließlich gleicht es einem Offenbarungseid, dass nicht etwa eine mit musikhistorischer und -bibliographischer Erfahrung, entsprechendem Personal und notwendiger Referenzliteratur ausgestattete Einrichtung ein „Musikverlagswiki“ ins Netz stellt, sondern (zweifellos engagierte) Studierende des Studiengangs Bibliotheks- und Informationswissenschaft der Leipziger Hochschule für Technik, Wirtschaft und Kultur in Verbindung mit der Internationalen Vereinigung der Musikbibliotheken (AIBM) und dem Leipziger Staatsarchiv auf die Idee kamen und hierfür verantwortlich zeichnen. ${ }^{19}$

15 Hierzu erfolgt bei Beer, Das Leipziger Bureau de Musique (wie Anm. 6), ein Versuch der Klärung.

16 Dass in den vergangenen Jahrzehnten auf diesem Gebiet nur wenig geschehen ist, belegt die (zweifellos in vieler Hinsicht willkommene) CD-ROM, die die zwischen 1971 und 1999 erschienene RISM-Reihe A/I (Einzeldrucke vor 1800) bündelt: Die in der Printversion vollzogene Anordnung undatierter Ausgaben eines Werks (teils willkürlich, teils alphabetisch nach Verlegern oder Verlagsorten) ist nun, sucht man nach Komponisten, durch eine rein alphabetische nach Titeln ersetzt - eine Hierarchisierung der Ausgaben ist noch immer nicht möglich, und somit bleibt etwa die nicht unwesentliche Frage nach Originalausgaben sowie der Chronologie von Titelauflagen und Nachdrucken unbeantwortet.

17 Vgl. Friedrich W. Riedel, „Die Arbeitsgemeinschaft für Geschichte der Musikpublikation“, in: Mf 18 (1965), S. 416-420 (mit einem Verzeichnis der Mitarbeiter, Publikationen und Arbeitsvorhaben). Friedrich Riedel teilte freundlicherweise mit, dass auf Grund „mangelnden Interesses die Angelegenheit allmählich eingeschlafen" sei. Glücklicherweise konnten die nach nicht langer Zeit in Vergessenheit geratenen Karteikästen „gerettet“ werden; sie befinden sich in der Obhut des Verfassers.

18 Music Publishing in Europe. Concepts and Issues. Bibliography, hrsg. von Rudolf Rasch (= The Circulation of Music 1), Berlin 2005, sowie Music Publishing in Europe. A Collection of Essays and Case Studies, hrsg. von dems. (= The Circulation of Music 2), Berlin 2008.

19 Es kann nicht verschwiegen werden, dass, obwohl die Initiative an sich zu begrüßen ist, die seit dem Spätsommer 2012 vorgelegten Ergebnisse kaum befriedigend sind, vor allem insofern, als alte Fehler auf Grund mangelnder Vertrautheit mit der Materie selbst und mit der Referenzliteratur weitergetragen werden. 
Von den bisher veröffentlichten Verlagsmonographien mit datierten (und hinsichtlich weiterer Gesichtspunkte annotierten) Listen der Gesamtproduktion - nach wie vor fehlen solche für Breitkopf \& Härtel, Schlesinger und Schott (ab 1798), um nur diese Firmen zu nennen - gehen nur wenige auf akademische Abschlussarbeiten oder sonstige Forschungen an musikwissenschaftlichen Facheinrichtungen zurück; ${ }^{20}$ die weitaus meisten Werke dieser Art entsprangen privatem Engagement: Genannt sei Alexander Weinmann, der, tätig als Musiker und Bibliothekar, bekanntlich Verzeichnisse der Verlagsproduktion sämtlicher im 18. und 19. Jahrhundert in Wien ansässigen Firmen vorlegte; Wolfgang Matthäus, der mit seiner Arbeit über die ersten Jahrzehnte des Verlags André in Offenbach Maßstäbe setzte, ${ }^{21}$ war Musiker; Hans Schneider, dem umfangreiche Monographien zu Heinrich Philipp Boßler, Makarius Falter und Johann Michael Götz zu verdanken sind, ${ }^{22}$ ist Musikverleger und -antiquar; erwähnt seien schließlich Hans Rheinfurth, der sein Buch über den Musikverlag Gombart $^{23}$ neben seiner Tätigkeit für RISM verfasste, und Thomas Emmerig, der sich als freier Autor den Regensburger Verlagshäusern zuwandte ${ }^{24}$ - sie alle folgten einem persönlichen Antrieb und einer individuellen Begeisterung für die Sache. Dies gilt ebenfalls für die gegenwärtig in Arbeit befindlichen Werke: Zu erwarten sind Monographien (soweit möglich mit kompletten datierten und annotierten Verzeichnissen unter Benutzung sämtlicher einschlägiger Quellen) über die Verlage von Friedrich Hofmeister in Leipzig, Heinrich Albert Probst in Leipzig und Nikolaus Simrock in Bonn; hiermit beschäftigt sind ein im Ruhestand befindlicher Musikwissenschaftler, ein Musiklehrer und ein musikologisch (auch als Verleger) ambitionierter Jurist, die sich von der längst aufgegebenen Hoffnung, irgendeine Förderung (abgesehen von wechselseitiger fachlicher und „logistischer" Unterstützung) zu erhalten, nicht entmutigen lassen und in mühseliger Kleinarbeit die weit ver-

20 Alexander Weinmann, Vollständiges Verlagsverzeichnis Artaria \& Comp. (= Beiträge zur Geschichte des Alt-Wiener Musikverlages 2/2), Wien 1952; Hans Rheinfurth, Der Musikverlag Lotter in Augsburg (ca. 1719-1845) (= Musikbibliographische Arbeiten 3), Münster 1976; Britta Constapel, Der Musikverlag Johann André in Offenbach am Main. Studien zur Verlagstätigkeit von Johann Anton André und Verzeichnis der Musikalien von 1800 bis 1840 (= Würzburger musikhistorische Beiträge 21), Tutzing 1998; Beate Martina Wollner [jetzt Leinhos], Carl Zulehner (1770-1841). Ein Musiker in Mainz (= Quellen und Abhandlungen zur Geschichte des Musikverlagswesens 4), Tutzing 2009. Übrigens unternahm Oskar Gutmann innerhalb seiner Dissertation Johann Karl Friedrich Rellstab. Ein Beitrag zur Musikgeschichte Berlins, Berlin 1910, erstmals (soweit zu sehen) den „Versuch eines Verlagsverzeichnisses“ (S. 145-177). Dass Hans-Christian Müllers Buch Bernhard Schott, Hofmusikstecher in Mainz. Die Frühgeschichte seines Musikverlages bis 1797. Mit einem Verzeichnis der Verlagswerke 1779-1797 (= Beiträge zur mittelrheinischen Musikgeschichte 16), Mainz 1977, als Hausarbeit zur Prüfung für den höheren Dienst an wissenschaftlichen Bibliotheken entstand, ist im vorliegenden Zusammenhang ebenfalls nicht unwesentlich.

21 Wolfgang Matthäus, Johann André Musikverlag zu Offenbach am Main. Verlagsgeschichte und Bibliographie 1772-1800, Tutzing 1973. Hingewiesen sei weiterhin auf Matthäus' Aufsatz „Der Musikverlag von Wolfgang Nikolaus Haueisen zu Frankfurt am Main“, in: Mf 22 (1969), S. 421-442.

22 Hans Schneider, Der Musikverleger Heinrich Philipp Bossler, Tutzing 1985; ders., Makarius Falter (1762-1843) und sein Münchner Musikverlag (1796-1888), Bd. 1, Tutzing 1993; ders., Der Musikverleger Johann Michael Götz (1740-1810) und seine kurfürstlich privilegirte Notenfabrique, 2 Bde., Tutzing 1989.

23 Hans Rheinfurth, Musikverlag Gombart Basel Augsburg (1789-1836), Tutzing 1999.

24 Thomas Emmerig, Regensburger Verlagsbuchhandlungen als Musikverlage (1750-1850) (= Quellen und Abhandlungen zur Geschichte des Musikverlagswesens 1), Tutzing 2000, sowie Regensburger Verlagsbuchhandlungen als Musikverlage (1850-1950) (= Quellen und Abhandlungen zur Geschichte des Musikverlagswesens 3), Tutzing 2007. 
streuten Fakten zusammentragen. ${ }^{25}$ Eine im Fach Musikwissenschaft geführte Diskussion über Desiderate, Standards, Forderungen und Notwendigkeiten hinsichtlich der Erstellung von Verlagsmonographien steht aus; entsprechend sind Stil, Herangehensweise und Zielsetzung bei den genannten Werken sehr unterschiedlich, was an dieser Stelle nicht vertieft sei. ${ }^{26}$ Nicht zu ersetzen sind monographische Darstellungen der genannten Art durch (an sich zweifellos verdienstliche) Publikationen, die das Phänomen Musikverlag und seine Geschichte durch die Nennung eines der musikalischen „Heroen“ gleichsam salonfähig machen. Ein „Begleitbuch zu einer Ausstellung“ über Beethoven und seinen Verlag Breitkopf \& Härtel $^{27}$ verfolgt naturgemäß zwar eine Vielzahl von Fragestellungen, versammelt anregende Beiträge, verfügt über eine bibliophile Ausstattung, zielt aber in seiner Intention mehr auf Beethoven als auf den Verlag - jener bleibt in der Darstellung im Grunde ohne Kontur, ohne Profil ${ }^{28}$ und auch ohne Konkurrenz, was wiederum einen Einblick in die Gesamtsituation, der Beethoven ebenso wie seine Kollegen unterworfen war, nicht ermöglicht. Abgesehen davon: Werke dieser Art, so willkommen sie sind, zeigen nur selten Auswirkungen in Form eines gesteigerten Interesses an der Materie; und die durchweg wohlwollenden Besprechungen der genannten verlagshistorischen Arbeiten weisen zudem kaum einen Ansatz zur Perspektivenbildung und Kontextualisierung des Gegenstands auf. ${ }^{29}$

Wie die vielen inzwischen zutage getretenen Zufallsfunde zeigen, ist es reizvoll, in zeitgenössischen Quellen, zumal in Verlagsarchiven und den dort lagernden Korrespondenzbeständen, nach Erwähnungen Beethovens und wessen auch immer zu suchen. Das eine oder andere willkommene, weil bisher unbekannte Detail tritt auf diese Weise mit Sicherheit ans Licht, doch zielt ein solches Verfahren im Grunde auf die fortdauernde Affirmierung des Kanons. Unberücksichtigt blieben dagegen die Vorgänge in ihrer Gesamtheit - etwa die Frage danach, wie ein Verlag sich angesichts der Konkurrenzsituation mit welchem Gesamtprogramm, welcher Ausstattung und welchen neuen Geschäftsideen positionierte, wie sich Präferenzen und Abneigungen der musikliebenden Öffentlichkeit in all ihren Wandlungen und Hintergründen widerspiegelten, welche Gattungen und Besetzungsformen bevorzugt wurden, inwieweit Mischkalkulation und Querfinanzierung betrieben wurden, um kostspielige Projekte „stemmen“ zu können, in welcher Weise Musiker und Komponisten („kleine“ wie „große") sich den Wünschen ihrer Verleger gegenüber verhielten und wie sich schließlich ein einzelnes Werk, sei es von Beethoven oder wem auch immer zu Papier gebracht, in diesem Gefüge sehen lässt. Um sich diesen und anderen Desideraten stellen zu können, bedarf es nicht nur sorgfältig erarbeiteter Dokumentationen der genannten Art, sondern auch eines profunden Überblicks (wenigstens in lexikalischer Form) über die gesamte Verlagslandschaft seit der Etablierung des selbständigen Musikverlagswesens in den 1770 er Jahren. Doch auch auf diesem Gebiet ist seit Robert Eitners vor über 100 Jahren

25 Nebenbei sei bemerkt, dass auch die nun nach rund 20 Jahren beendete Arbeit des Verfassers über das Leipziger Bureau de Musique (s. Anm. 6) als „Privatvergnügen“ eingestuft werden kann.

26 Vgl. hierzu Beer, Das Leipziger Bureau de Musique (wie Anm. 6), Einleitung.

27 Gemeint ist Beethoven und der Leipziger Musikverlag Breitkopf \& Härtel, hrsg. von Nicole Kämpken und Michael Ladenburger, Bonn 2007.

28 Vgl. hierzu weiter unten, S. $381 f$.

29 Aus Raumgründen kann an dieser Stelle auf die Rezensionen in der Musikforschung, wo übrigens in den vergangenen vierzig Jahren nicht einmal eine Handvoll Beiträge zu jener Thematik erschien, nicht näher eingegangen werden. 
vorgelegtem Verzeichnis kaum etwas geschehen ${ }^{30}$ - sieht man einmal ab von Otto Erich Deutschs nach wie vor als Notbehelf dienenden und zu manchen Missverständnissen und Fehlinterpretationen verführenden, gerade einmal 32 Seiten umfassenden und einen mehr als 50 Jahre zurückliegenden Forschungsstand präsentierenden Heftchen, das 40 vorwiegend deutsche und österreichische Verlage aufzählt und Hilfen zur Datierung nach Verlagsnummern bietet; ${ }^{31}$ überdies wird die gegenwärtige Situation anschaulich anhand des im jüngst erschienenen neuen Beethoven-Werkverzeichnis dokumentierten bruchstückhaften Kenntnisstands. ${ }^{32}$ Für Frankreich, Italien, Dänemark und England ist der Befund hingegen erfreulicher: Nachschlagewerke und allgemeine Darstellungen erleichtern die Arbeit und vor allem den grundlegenden Zugang zur Materie ${ }^{33}$ - bezogen auf den deutschsprachigen Raum kann einstweilen nur von Planungen die Rede sein. ${ }^{34}$ Auch bezüglich anderer Aspekte, genannt seien die Dokumentation der Pariser Presseanzeigen des 18. Jahrhunderts oder der Londoner Stationers' Hall-Einträge, zeigen sich im Ausland erfreuliche Entwicklungen. ${ }^{35}$ Es ist geradezu symptomatisch, dass Kurt Dorfmüller und Gertraut Haberkamp die von Liesbeth Weinhold und Alexander Weinmann (beide in den 1980er-Jahren verstorben) hinterlassenen Materialien zu Verleger- und Händlerkatalogen im deutschsprachigen Raum erst 1995 herausgeben konnten ${ }^{36}$ - ob jemals eine systematische Befragung und Auswertung der bibliographierten (und inzwischen durch zahlreiche Ergänzungen zu bereichernden) Quellen erfolgen wird, muss offenbleiben; dies gilt auch für Verlagsanzeigen in unterschiedlichen Periodika. Allerdings sollte die Forschung, sofern sie sich einmal gezielt der Sache annimmt, nicht dabei stehenbleiben, das überaus umfangreiche Material wiederum lediglich auf einzelne Komponisten abzuklopfen ${ }^{37}$ - ist ein Name abgearbeitet, so wird man sich mit derselben Intention einem anderen zuwenden können und neben Verlags- und Händlerkatalogen erneut Hunderte von Jahrgängen etlicher Dutzend Zeitungen und Zeitschriften (die, nebenbei bemerkt, nur in Ausnahmefällen digitalisiert sind) durchblättern müssen. Trotz der zweifellos zu erwartenden immensen Materialfülle bliebe

30 Robert Eitner, Buch- und Musikalien-Händler, Buch-und Musikaliendrucker nebst Notenstecher, nur die Musik betreffend, nach den Originaldrucken verzeichnet, Leipzig 1904. Vgl. auch Axel Beer, Musik zwischen Komponist, Verlag und Publikum (wie Anm. 6), S. 47-96.

31 Otto Erich Deutsch, Musikverlagsnummern. Eine Auswahl von 40 datierten Listen 1710-1900, Berlin 1961 (erweiterte Fassung der 1946 in London erschienenen Music Publisher's Numbers).

32 Kurt Dorfmüller, Norbert Gertsch und Julia Ronge (Bearb.) unter Mitarbeit von Gertraut Haberkamp und dem Beethoven-Haus Bonn, Ludwig van Beethoven. Thematisch-bibliographisches Werkverzeichnis, revidierte und wesentlich erweiterte Neuausgabe des Verzeichnisses von Georg Kinsky und Hans Halm, Bd. 2, München 2014, S. 733-786 (Kapitel Verlage). Ein größeres Bemühen um die Bündelung von Fachwissen hätte der Sache (nicht nur bezogen auf den Komponisten) sicherlich genutzt.

33 Anik Devriès und François Lesure, Dictionnaire des éditeurs de musique français, 2 Bde., Genf 1979 und 1988; Bianca Maria Antolini, Dizionario degli editori musicali italiani 1750-1930, Pisa 2000; Dan Fog, Musikhandel og Nodetryk i Danmark efter 1750, 2 Bde., Kopenhagen 1984; Charles Humphries und William C. Smith, Music Publishing in the British Isles, London 1954. Für Russland existiert eine knappe Übersicht von Cecil Hopkinson, Notes on Russian Music Publishers, Bath 1959.

34 Ein Lexikon der deutschen Musikverlage für den Zeitraum bis ca. 1830 ist vom Verfasser dieser Zeilen zumindest angedacht.

35 Anik Devriès-Lesure, L'édition musicale dans la presse parisienne au XVIII siècle. Catalogue des annonces, Paris 2005; Michael Kassler, Music Entries at Stationers' Hall 1710-1818, Aldershot 2004.

36 Liesbeth Weinhold und Alexander Weinmann, Kataloge von Musikverlegern und Musikalienhändlern im deutschsprachigen Raum 1700-1850 (= Catalogus musicus 15), Kassel u. a. 1995.

37 Vgl. Gertraut Haberkamp, Anzeigen und Rezensionen von Mozart-Drucken in Zeitungen und Zeitschriften, erschienen in mittlerweile 19 Folgen in den Mozart Studien (Bd. 1, 1992, bis Bd. 21, 2012). 
das Ergebnis im Grunde mager, da auf diese Weise vorgelegte Fakten ohne Relation sind, der sie aber natürlich bedürfen, um überhaupt Fragestellungen und Erkenntniswünsche zulassen zu können. Im Übrigen kann es auch nicht darum gehen, weitere Datenbankleichen zu produzieren.

Das häufige Fehlen bibliographisch gesicherter sowie durch Vergleichsmöglichkeiten und notwendige Kontextualisierung benutzbarer Faktengrundlagen führt ebenso wie die Tatsache, dass einschlägige Forschungsarbeiten, wie oben dargelegt, lediglich „Privatvergnügen“ auf jenem Gebiet ausgewiesener Fachleute bzw., und im krassen Gegensatz dazu, hier und da einem bedenklichen Dilettantismus ausgesetzt sind, zwangsläufig zur Marginalisierung des Gegenstands, zu Irrtümern und unangemessenen Pauschaldeutungen. Beispiele wurden gegeben, und es zeigt sich, dass gerade jene Verallgemeinerungen, nicht selten vollzogen im vollkommen quellenfreien Raum, eine beträchtliche Lebenserwartung besitzen. Sie lassen sich auf Grund ihrer Kompaktheit bequem weitertragen, beinhalten keinen unmittelbar erkennbaren Widerspruch und fügen sich bruchlos in den jeweiligen, oft genug den Kanon affirmierenden und ästhetisch begründeten Argumentationsstrang: Die Behauptung etwa, dass der Beethoven-Verlag Breitkopf \& Härtel „hinsichtlich seines Verlagsprogramms stets ambitioniert" war, ${ }^{38}$ mag nicht falsch sein; ob allerdings vor der Niederschrift jener wenigen Worte die an sich notwendige, eine differenzierte Aussage überhaupt ermöglichende sorgfältige Betrachtung des Verlagsprogramms (der Forschung steht ein datiertes und kommentiertes Verzeichnis nach wie vor nicht zur Verfügung) erfolgte, bleibt offen. Was zudem „ambitioniert“ eigentlich heißen soll und worauf sich die Ambition bezieht, wird, sofern nicht einfach die durch die Nennung Beethovens bereits implizite ästhetische Ebene angesprochen ist, ebenso wenig klar. Hierzu nur einige wenige Beobachtungen, die die Dimension etwaiger Fragestellungen umreißen mögen: In der Nummernfolge der verlagsinternen und sich in Form der Plattennummern auf den Druckausgaben niederschlagenden Buchführung figurieren bei Breitkopf \& Härtel vor Beethovens Opera 69 und 67 (in dieser Reihenfolge; Verlagsnummern 1328 und 1329) die Violin- und Flötenduos op. 15-17 von (bösartig könnte man formulieren: eines gewissen) Johann Friedrich Barmann; auf Beethoven folgen Werke von Franz Xaver Mozart, Steibelt und Wiedebein. Während letztere Namen seinerzeit zum Teil jedenfalls eine gewisse bis erhebliche Bekanntheit besaßen, die gegenwärtig noch nicht vollkommen verblasst ist, attestiert die alte $M G G$ (die neue enthält bezeichnenderweise keinen diesbezüglichen Eintrag) der Musik jenes Barmann, sie sei „nicht von großer Einfallsgabe“ geprägt, schickt aber hinterher, dass sie „in ihrer Zeit sehr günstig aufgenommen“ worden sei. ${ }^{39}$ Sollte die Beurteilung hinsichtlich der mangelnden Einfallsgabe zutreffen, so würde dies freilich einen recht auffälligen Gegensatz zu dem bedeuten, was man Beethovens Musik zu unterstellen gewohnt war. Wichtiger aber ist der Hinweis auf die günstige Aufnahme, den der Verfasser des Artikels nur aus den Rezensionen der Allgemeinen musikalischen Zeitung gezogen haben kann, da sich anderswo nichts dieser Art findet. ${ }^{40}$ Dass allerdings die Flötenduos op. 16, da sie als „angenehm und dem Instrumente angemessen“ beurteilt wurden, „den Liebhabern mit Fug u. Recht zu empfehlen“ waren, ${ }^{41}$ darf nicht als Reflex der Beliebtheit (eine solche

38 Beethoven und der Leipziger Musikverlag Breitkopf \& Härtel (wie Anm. 27), S. V.

39 Albert Vander Linden, Artikel „Barmann“, in: MGG1, Bd. 15, Kassel u. a. 1973, Sp. 483.

40 In Axel Beer, „Empfehlenswerthe Musikalien“. Besprechungen musikalischer Neuerscheinungen außerhalb der Fachpresse (Deutschland, 1. Hälfte des 19. Jahrhunderts), 2 Bde., Göttingen u. a. 2000 und 2001, ist keine Besprechung Barmann'scher Werke verzeichnet.

41 AmZ 11, Nr. 52, 27. Sept. 1809, Sp. 848. 
konnte sich noch gar nicht eingestellt haben) interpretiert werden, sondern als reine Werbemaßnahme im verlagseigenen Organ, das - zweifellos nicht zufällig - erst drei Wochen später eine Anzeige des jüngst erschienenen Werks in seinem Intelligenzblatt einrückte. ${ }^{42}$ Jene Anzeige enthält neben anderen Titeln auch Beethovens Opera 67-70, von denen die ersten drei schon im April erstmals annonciert worden waren, ${ }^{43}$ und findet sich zudem bezeichnend für die Streuung der Information - auch in der überregional verbreiteten Berliner, Breslauer, Gothaer, Hamburger und Leipziger Tagespresse. ${ }^{44}$ Barmanns Musik sollte und musste sich also gut verkaufen; sie erbrachte, sofern die erkennbaren Werbestrategien Erfolg zeigten, im Verbund mit ähnlicher Ware die finanziellen Grundlagen für die hinsichtlich der Honorare und der Produktion besonders kostspieligen Beethovenausgaben, deren günstige Aufnahme in der Öffentlichkeit (gemessen an Verkaufszahlen und anders als Barmanns Musik) sich auf Grund des hohen musikalischen Anspruchs nicht vorhersagen, geschweige denn durch die Anpreisung ihrer „angenehmen“ und den Liebhabern willkommenen Eigenschaften steuern ließ. Folglich enthält die bekannte Rezension der Fünften Sinfonie Beethovens durch E. T. A. Hoffmann in Härtels Fachblatt nichts davon kein Wort zur Markttauglichkeit, sondern eine in wohlklingende Bildersprache verpackte Einführung in das „Wesen der Romantik“, 45 und - dies sei noch bemerkt - sicherlich ging es hierbei allenfalls zweitrangig um die Dokumentation musikästhetischen Denkens (so gerne man immer wieder aus dem Text zitiert), sondern erneut um eine allerdings in dieser Art bisher nicht dagewesene Werbemaßnahme, die die Tatsache, dass erstmals eine Sinfonie des Meisters bei Breitkopf \& Härtel erschienen war, gleichsam mit einem literarischen Paukenschlag hervorheben sollte. Gewiss zeigt das Verlagsprogramm Ambitioniertheit, aber eine solche, die auf marktstrategische Erwägungen und daraus folgende Notwendigkeiten zielt, nicht dagegen im Sinne einer durchgängig zu beobachtenden Bestätigung später gewachsener ästhetischer Normen zu verstehen ist. Die Inverlagnahme von Beethovens Musik war für Breitkopf \& Härtel in erster Linie ein Renommierprojekt, das der Firma, eine geschickte Querfinanzierung vorausgesetzt, nicht unwesentliche Vorteile gegenüber der Konkurrenz einbrachte. Dass diese Beobachtung keineswegs gegen den Verlag spricht, sondern für seine Professionalität, sei betont. Wir müssen uns nur daran gewöhnen, auch wenn es um Beethoven geht - schließlich wandte dieser sich der Firma bewusst zu; und wir müssen uns auch daran gewöhnen, dass vor dem Hintergrund des Gesagten der Musik eines Barmann ein gewisses Maß an kulturgeschichtlicher Bedeutung zukommt.

Wenn von „erbärmlichem Notenstich“ - ein zweites Beispiel für eine Formulierung sei gegeben, die man zunächst ohne weiteres Nachdenken hinnehmen mag - die Rede ist, wird man vielleicht umso mehr dem Urheber jener Aussage blindes Vertrauen schenken, als ein Werk Mozarts betroffen ist und ein Verleger bzw. Notenstecher attackiert wird, den kaum ein Mensch kennt. Die Botschaft - ein verantwortungsloser Pfuscher, der sich an einer Schöpfung des Meisters vergreift - ist unmissverständlich, und so erübrigten sich weitere

$42 \operatorname{AmZ}$ 12, Intelligenzblatt 1 zu Nr. 3, 18. Okt. 1809.

$43 \mathrm{AmZ}$ 11, Intelligenzblatt 8 zu Nr. 29, 19. Apr. 1809.

44 Berlinische Nachrichten von Staats- und gelehrten Sachen, Beilage zu Nr. 131, 2. Nov. 1809; Schlesische privilegirte Zeitung (Breslau) Nr. 130, 4. Nov. 1809; Allgemeiner Anzeiger der Deutschen (Gotha) Nr. 271, 8. Okt. 1809; Staats- und Gelehrte Zeitung des Hamburgischen unpartheyischen Correspondenten, Beilage zu Nr. 160, 7. Okt. 1809; Leipziger Zeitung, Beilage zu Nr. 189, 27. Sept. 1809.

45 „Sinfonie pour 2 Violons [...]" (Rezension), in: $A m Z$ 12, Nr. 40, 7. Juli 1810, Sp. 630-642, und Nr. 41, 11. Juli 1810, Sp. 652-659, hier: Sp. 633. 
Nachforschungen. ${ }^{46}$ Man wusste also über Philipp Jakob von Thonus, so der Name des Gescholtenen, bislang nichts. Inzwischen konnte neben seiner Lebenszeit (1748-1799) eine Reihe von Fakten erhoben werden, die eine schlagwortartige Abwertung zumindest nicht mehr zulassen - hierzu nur so viel: ${ }^{47}$ Für Constanze Mozart war von Thonus, als sie im Herbst 1795 nach Leipzig kam, ein nicht unwichtiger, zweifellos schon zuvor kontaktierter Ansprechpartner, bei dem sie im Blick auf ihr ungewöhnliches Vorhaben, durch das Verlegen unbekannter Werke eines Verstorbenen Gewinn zu erzielen, professionelle und verlässliche Unterstützung fand. Von Thonus stach für sie nicht nur das Klavierkonzert KV 503 - hiermit wollte man eine Serie der nachgelassenen Arbeiten eröffnen ${ }^{48}$-, sondern war auch an weiteren Projekten beteiligt: Der vom damaligen Leipziger Nikolai-Organisten und späteren Thomaskantor August Eberhard Müller verfasste und bei Günther \& Böhme in Hamburg (beide hatten sich kurz zuvor von Leipzig aus dort angesiedelt) erschienene Klavierauszug von La clemenza di Tito ${ }^{49}$ zeigt ebenso das unverkennbare Thonus-Stichbild wie die gleichfalls aus Müllers Feder stammende, von Schmiedt \& Rau in Leipzig verlegte Anweisung zum genauen Vortrage der Mozartschen Clavierconcerte. ${ }^{50}$ Augenscheinlich agierte von Thonus als Teil eines von Constanze Mozart intendierten Beziehungsgeflechts, in dem Stümper nichts zu suchen hatten: Müllers Expertenschaft war unbestritten, und von Thonus, der seit mittlerweile fast zwei Jahrzehnten im Leipziger Musikleben verwurzelt war und auch Aufträge von außerhalb erhielt, galt zumindest einem der Zeitgenossen, die von der Materie etwas verstanden, als (so ändern sich die Anschauungen!) einer „unsrer geschicktesten Notenstecher". 51 Der Eintritt Gottfried Christoph Härtels in das Haus Breitkopf Ende des Jahres 1795 eröffnete für Constanze Mozart zusätzliche Perspektiven, ohne dass sie einen Grund sah, sich von Philipp Jakob von Thonus abzuwenden, der seine eigenen Verlagsprodukte seitdem bei der alles beherrschenden Firma in Kommission erschienen ließ. Unter ihnen befinden sich wiederum Werke Mozarts: neben der (Teil-)Erstausgabe der Gärtnerin aus Liebe auch einige noch immer rätselhafte Arrangements und Mozart zugeschriebene Werke, über deren Herkunft - auf jeden Fall spielte August Eberhard Müller wiederum eine nicht unwesentliche Rolle - möglicherweise aus dem „Dunstkreis“ der Wit-

46 Solche wurden auch nicht angestellt, nachdem das herbe Urteil aus dem Köchelverzeichnis (es findet sich in der 3. Auflage zu KV 503, s. KV 3 , S. 640) verschwunden war.

47 Ausführlicher bei Beer, Das Leipziger Bureau de Musique (wie Anm. 6).

48 Das Titelblatt trägt den Vermerk „No 1. del retaggio del defunto [...] “. Pränumerationsanzeige vom 16. Nov. 1795 erstmals in: Leipziger Zeitungen Nr. 234, 30. Nov. 1795, S. 1678; früheste Anzeige nach Fertigstellung: Berlinische Nachrichten von Staats- und gelehrten Sachen Nr. 126, 20. Okt. 1796 (bei Concha \& Francke zu haben). Gertraut Haberkamp, Die Erstdrucke der Werke von Wolfgang Amadeus Mozart, Tutzing 1986, Textband, S. 274, notiert als früheste Anzeige den 21. Juni 1797 im Hamburgischen Correspondenten (Nr. 98).

49 Pränumerationsanzeige Constanze Mozarts zu La clemenza di Tito (datiert Mai 1795) zuerst in der Staats- und gelehrten Zeitung des Hamburgischen unpartheyischen Correspondenten, Beilage zu Nr. 92, 10. Juni 1795.

50 Vgl. hierzu Axel Beer, „Musikverlag und Musikalienhandel in Leipzig in der 2. Hälfte des 18. Jahrhunderts“, sowie ders., „Verlagsforschung in Leipzig - Desiderate und Perspektiven“, in: Stefan Keym und Peter Schmitz (Hrsg.), Das Leipziger Musikverlagswesen. Innerstädtische Netzwerke und internationale Ausstrablung (Druck in Vorbereitung).

51 Friedrich Justin Bertuch, Vorrede zu Anton Schweitzers Polyxena ein lyrisches Melodram, Weimar 1793. Von Thonus' Name ist zwar nicht genannt, doch geht - abgesehen vom unverwechselbaren Stichbild auch aus der Korrespondenz zwischen ihm und Bertuch hervor, dass er den Auftrag ausgeführt hat (vgl. von Thonus an Bertuch (Weimar), Leipzig 10. Febr. 1793 und 18. März 1793, Goethe- und SchillerArchiv Weimar, GSA 6/1934). 
we einmal genauer nachzudenken sich vor dem geschilderten Hintergrund sicher lohnen würde. ${ }^{52}$

Selbstverständlich dient das genannte Beispiel nicht der gängigen Strategie, die Befassung mit einer wenig bekannten Person durch die Nennung eines geläufigen Namens zu rechtfertigen, sondern es mag zeigen, in welchem Maße die (vermeintlich sogar begründete) Marginalisierung unwichtig erscheinender Fakten einem beträchtlichen Erkenntnisgewinn (hier immerhin bezüglich der Rezeptionsgeschichte Mozarts) im Wege stehen kann. Die stetig aufs Neue aufkeimende und keineswegs unnütze Diskussion über den Gegenstand des Fachs Musikwissenschaft, seine Grenzen und seine Methoden sowie über den Grad der Flexibilität angesichts vielfältiger neuer Herausforderungen umgeht in auffälliger Weise die Frage nach dem Wert und der Perspektive einer - nota bene - sich nicht selbst genügenden Grundlagenforschung. So besitzt die vor kurzem ausgesprochene These, dass die "Sinngebung" des Fachs mit einem "datensammelnden Positivismus" nicht in Einklang zu bringen sei, ${ }^{53}$ ein gewisses $\mathrm{Maß}$ an Repräsentativität: Dort, wo es um Ästhetik, Hermeneutik und Epistemologie geht, um das Erkennen und Deuten komplexer musikalischer Strukturen, um das Entfalten, Rechtfertigen und Diskutieren vielgestaltiger Theorien, zudem um die Anschlussfähigkeit des Fachs an Denkmodelle benachbarter Geistes- und auch Sozialwissenschaften, hat offenbar eine faktenorientierte Erfassung, Erschließung und Auswertung umfangreicher Quellenressourcen keinen Platz, ${ }^{54}$ selbst wenn dies mancherlei Präzisierungen, unverbrauchte Fragestellungen und einen Anstoß zur Bereicherung des Methodenschatzes beitragen könnte. Natürlich spricht hier der Historiker, der es gewohnt ist, sich seinem Gegenstand über die Zeiten hinweg anzunähern und ihn nicht - das wäre das Gegenmodell - aus seiner Zeit zu befreien und all das von ihm abzuschütteln, dessen Berücksichtigung mit dem Stigma des biederen Positivismus gezeichnet werden könnte.

Sich auch ohne historische Ambition mit Musik zu befassen, ist selbstverständlich legitim; sofern aber der eigentliche Grund hierfür darin besteht, dass der Zugang zu vielfältigem Quellenmaterial durch nur schwer zu überwindende Hindernisse versperrt ist, gerät man in Gefahr, Ursache und Wirkung zu vertauschen. Dass es bereits Geisteswissenschaftler gibt, die das Lesen von Frakturschrift ausgesprochen unvergnüglich finden und um Kurrentschriften einen großen Bogen machen, ist nicht mehr zu verdrängen. Bezeichnend ist eine Aussage in der 2014 erschienenen Einführung in das musikwissenschaftliche Arbeiten: Danach wird man ein „Archiv oder ein Museum [!] [...] erst aufsuchen müssen [!], wenn man sich in einer Abschlussarbeit oder Dissertation für speziellere Fragestellungen

52 Es handelt sich um die Verlagsnummern 24-29 des Leipziger Bureau de Musique, das nach von Thonus' Tod den Verlag von den Erben übernommen und in das eigene Programm integriert hatte; vgl. Beer, Das Leipziger Bureau de Musique (wie Anm. 6). Constanze Mozart stand noch Ende des Jahres 1798 mit von Thonus in Verbindung; vgl. ihren Brief an Breitkopf \& Härtel, Wien 16. Nov. 1798 (Mozart. Briefe und Aufzeichnungen. Gesamtausgabe (wie Anm. 11), Band 4, S. 220).

53 Michael Walter, „Musikwissenschaft und ihr Gegenstand“, in: AfMw 69 (2012), S. 293-303, hier: S. 303.

54 Auch der jüngste, inhaltlich weit ausholende Beitrag zur Diskussion (Historische Musikwissenschaft. Grundlagen und Perspektiven, hrsg. von Michele Calella und Nikolaus Urbanek, Stuttgart/Weimar 2013) kann nicht als Ermunterung verstanden werden, Grundlagenforschung oder gar verlagshistorische Untersuchungen (Namen von Musikverlegern weist das Register nicht auf - an der Spitze rangieren einmal mehr Adorno, Beethoven und Dahlhaus) und hierzu notwendige Quellenstudien zu betreiben. 
interessiert." ${ }^{55}$ Bei welcher Gelegenheit die Studierenden eigentlich den Umgang mit Archivgut erlernt und geübt haben sollen, wird nicht thematisiert, und die Zahl derjenigen, die jenen Umgang gewohnt sind und die Resultate (natürlich nicht mit dem Ziel, „datensammelnden Positivismus“ zum Prinzip zu erheben) wie auch den Weg zu ihnen in die universitäre Lehre integrieren, wird in absehbarer Zeit bedenklich zurückgehen. ${ }^{56}$ Auch fällt es nicht leicht, mit sinnvollen Argumenten den Studierenden klarzumachen, dass eine Arbeit dieser Art zwar (selbstverständlich verbunden mit übergeordneten Fragestellungen und Perspektiven) wissenschaftlichen Gewinn erbringen kann, aber üblicherweise außerhalb des fachlichen Organisationsgefüges bzw. inhaltlichen „mainstreams“ angesiedelt ist. Gleichfalls ist es schwer zu vermitteln, dass etwa das immense Quellenmaterial zur Geschichte des Musikverlagswesens in der Regel nur - der Vergleich mit den Rosinen im Kuchen ist kaum zu umgehen - auf der Suche nach bestimmten, zumeist bekannten Namen durchforstet wird. Der historische „Abraum“ bleibt hierbei ungenutzt liegen, sei es deshalb (wobei nicht ausgeschlossen sei, dass auch andere Ursachen ins Feld geführt werden können), weil er aus ästhetischer Sicht als unbedeutend angesehen wird, sei es, weil man befürchtet, bei genauerem Hinsehen bewährte Theorien ins Wanken zu bringen, oder sei es, weil man angesichts der Fülle (oder aus soeben erwähnten Gründen) schlicht und einfach kapituliert. Sich letzteres einzugestehen, wirft freilich Probleme auf; leichter ist es, mit dem Begriff Bedeutung und dessen Gegenteil zu argumentieren. Eine Disposition wie diese ist - mutatis mutandis - nicht weit entfernt von der oft genug gescholtenen Wahrnehmungsweise wenig oder gar nicht reflektierender Musikrezipienten, die die Werke Beethovens oder wessen auch immer wegen ihrer eingängigen Melodien schätzen und eine Sonate des Meisters als Potpourri der schönen Stellen zu hören gewohnt sind. Wenn Beethoven den Kampf gegen die Musikverleger gewonnen hat, wäre er denn letztlich auch im Kampf gegen die Sonatenhauptsatzform siegreich geblieben - natürlich ist beides Unsinn.

55 Matthew Gardner und Sara Springfeld, Musikwissenschaftliches Arbeiten. Eine Einführung, Kassel u. a. 2014, S. 162. Dass im Übrigen die Informationen zu älteren Musikdrucken und Drucktechniken (S. 90f.) sowie zu Datierung und Verzeichnung älterer Musikalien (S. 125f. und 202f.) unzulänglich und bisweilen fehlerhaft sind, kann nicht verschwiegen werden.

56 Der Verfasser dieser Zeilen bemüht sich, neben Lehrveranstaltungen zur Geschichte des Musikverlagswesens auch Übungen zu Paläographie und Quellenkunde möglichst regelmäßig anzubieten. 